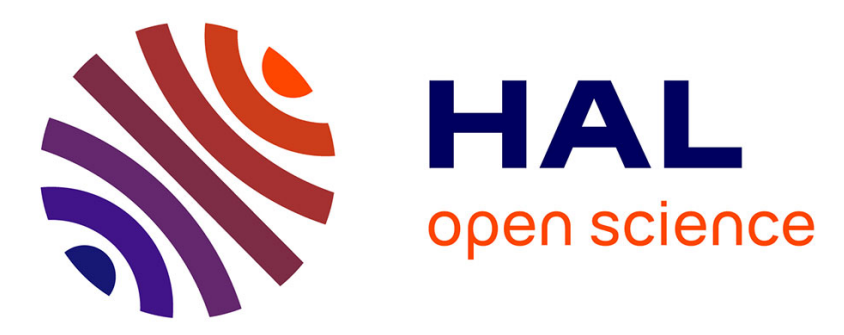

\title{
MULTI-SPECTRAL IMAGE ANALYSIS FOR SKIN PIGMENTATION CLASSIFICATION
}

Sylvain Prigent, Xavier Descombes, Didier Zugaj, Philippe Martel, Josiane Zerubia

\section{- To cite this version:}

Sylvain Prigent, Xavier Descombes, Didier Zugaj, Philippe Martel, Josiane Zerubia. MULTISPECTRAL IMAGE ANALYSIS FOR SKIN PIGMENTATION CLASSIFICATION. IEEE International Conference on Image Processing (ICIP), Sep 2010, Hong Kong, China. inria-00499492

\section{HAL Id: inria-00499492 \\ https://hal.inria.fr/inria-00499492}

Submitted on 9 Jul 2010

HAL is a multi-disciplinary open access archive for the deposit and dissemination of scientific research documents, whether they are published or not. The documents may come from teaching and research institutions in France or abroad, or from public or private research centers.
L'archive ouverte pluridisciplinaire $\mathbf{H A L}$, est destinée au dépôt et à la diffusion de documents scientifiques de niveau recherche, publiés ou non, émanant des établissements d'enseignement et de recherche français ou étrangers, des laboratoires publics ou privés. 


\title{
MULTI-SPECTRAL IMAGE ANALYSIS FOR SKIN PIGMENTATION CLASSIFICATION
}

\author{
Sylvain Prigent ${ }^{1}$, Xavier Descombes $^{1}$, Didier Zugaj ${ }^{2}$, Philippe Martel ${ }^{2}$, Josiane Zerubia ${ }^{1}$ \\ ${ }^{1}$ EPI Ariana INRIA/I3S, 2004 route des Lucioles, BP93 06902 Sophia Antipolis, Cedex. France \\ email: Sylvain.Prigent@inria.fr, Xavier.Descombes@inria.fr, Josiane.Zerubia@inria.fr \\ ${ }^{2}$ Galderma R\&D, 2400 Route des Colles, BP87, 06902 Sophia-Antipolis, Cedex. France \\ email: Didier.Zugaj@galderma.com, Philippe.Martel@galderma.com
}

\begin{abstract}
In this paper, we compare two different approaches for semiautomatic detection of skin hyper-pigmentation on multispectral images. These two methods are support vector machine (SVM) and blind source separation. To apply SVM, a dimension reduction method adapted to data classification is proposed. It allows to improve the quality of SVM classification as well as to have reasonable computation time. For the blind source separation approach we show that, using independent component analysis, it is possible to extract a relevant cartography of skin pigmentation.
\end{abstract}

Index Terms - skin hyper-pigmentation, multi-spectral images, support vector machine, independent component analysis, data reduction.

\section{INTRODUCTION}

An accurate quantification of skin diseases is important both for quantifying the degree of the pathology, and evaluating as early as possible, the efficiency of a treatment. In this paper, we propose to use multi-spectral and hyper-spectral images to analyse hyper-pigmentation of the skin. The interest of working with multi-spectral images is to have a more accurate information on skin properties than those obtained on conventional cameras. Several methods based on absorbance spectrum in visible and near infra-red have already been presented in the literature for quantifying the melanin in the skin $[1,2]$. The main idea of these methods is to select specific spectral bands in the data in order to extract information on skin pigmentation. One of the most famous algorithms has been proposed by Stamatas in [1]. This algorithm is based on the analysis of the skin chromophores absorbance spectrum. An affine model of the melanin absorbance is proposed in the spectrum range of $630-700 \mathrm{~nm}$ :

$$
A_{m}(\lambda)=a \lambda+b,
$$

where $A_{m}$ represents melanin absorbance, $a$ and $b$ are linear coefficients and $\lambda$ the wavelength. The spectrum can be corrected by substracting the influence of melanin:

$$
A_{c}(\lambda)=A(\lambda)-A_{m}(\lambda)
$$

Concentrations of oxy-hemoglobin and deoxy-hemoglobin are calculated by solving the system given by the BeerLamber law applied to two specified wavelengths $\left(\lambda_{1}=560\right.$ $\left.\mathrm{nm}, \lambda_{2}=580 \mathrm{~nm}\right)$ :

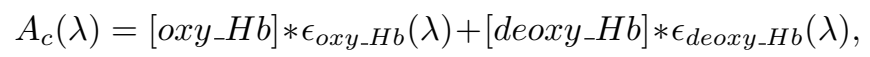

where $\epsilon_{O x y_{-} H b}$ and $\epsilon_{\text {deoxy_Hb}}$ represent extinction coefficients which have tabulated values. Finally, corrected melanin concentration cartography can be extracted by correcting the affine estimation of melanin concentration taking into account the deoxy-hemoglobin influence in the 630-700 nm range:

$$
\begin{aligned}
& {[\text { Melanin }]_{c}=[\text { Melanin }]} \\
& \quad-\left([\text { deoxy_Hb }] * a\left[\epsilon_{\text {deoxy_Hb }}\right]+b\left[\epsilon_{\text {deoxy_Hb}}\right]\right),
\end{aligned}
$$

where $a\left[\epsilon_{\text {deoxy_Hb }}\right]$ and $b\left[\epsilon_{\text {deoxy_Hb }}\right]$ are coefficients in the linear model of the deoxy-hemoglobin absorbance in the range $630-700 \mathrm{~nm}$.

In this paper, we propose to extract melanin component considering the whole spectrum. Two methods are proposed. The first one is based on support vector machines (SVMs) with a preprocessing dimension reduction step. The second method quantifies the melanin component assuming that the multispectral image is a linear mixture of components constituting the skin. SVMs are considered as one of the most efficient methods to classify multi and hyper-spectral images. SVM computs a class separator from learning pixels in order to classify all the data. For hyper-spectral images, SVMs are often associated with dimension reduction algorithms [3], such as principal component analysis (PCA) or projection pursuit [4], in order to avoid the Hughes phenomenon [5]. In this paper, a projection pursuit method has been chosen since it permits dimension reduction based on an index $I$ optimizing the classification. As an unmixing method we propose to use independent component analysis (ICA).

The paper is organized as follows. In section 2 we present the projection pursuit algorithm and classification by SVM. In section 3 we compare several ICA methods. In section 4 we report obtained results. In section 5 conclusions are drawn. 


\section{PROJECTION PURSUIT WITH SVM}

\subsection{Projection pursuit}

The common way proposed in the literature to select spectral bands for skin analysis is to do it manually. Here, we propose to employ projection pursuit. Projection pursuit, originally proposed in [4], can provide physics spectral analysis involving the whole spectrum. It consists of two steps. First the spectrum is partitioned into groups of adjacent bands, and then each group is projected in a unique vector. Thus, a reduced space is obtained and its size is equal to the number of groups. Each group projection vector has to be simultaneously representative of its corresponding group and as "far" as possible from the other vectors to assure band separability in reduced data space. For this purpose, an index $I$ is defined to optimize the projection vector basis. The choice of $I$ defines the optimal subspace and can be adapted to targeted applications. Along the classical projection pursuit process, the index is iteratively maximized on the projection vectors. In our application, projection pursuit is used as a preprocessing step for classification. That is why, as proposed in [6], we prefer to calculate the index $I$ on the training classes. This is also significantly faster than to consider the whole volume.

\subsubsection{Choice of the projection index}

In the literature, the most common projection pursuit indices are Bhattacharyya and Kullback-Leibler distances [7]. For classification purpose more specific indices can be used. For example, an index based on histogram analysis is proposed in [8]. On the other hand, such indices require manual parameter specification, and don't improve significantly the classification accuracy. That is why we prefer to use the KullbackLeibler distance $D(i, j)=0.5(H(i, j)+H(j, i))$, where:

$$
H(i, j)=\int f_{i}(x) \ln \frac{f_{i}(x)}{f_{j}(x)} d x,
$$

and $f_{i}, f_{j}$ are the distributions of the two training classes.

\subsubsection{Spectrum partitioning}

The second important step in the dimension reduction is to choose a reasonable partitioning of the spectrum into groups. In the original projection pursuit [4], the spectrum is separated in $K$ groups having the same number of bands. Since the purpose is to study information about skin components, it seems to be judicious to select a partitioning adapted to the skin properties. In [7], a partitioning method based on the index $I$ is proposed. If $K$ is a specification parameter, the method partitions the spectrum as follows: first, the spectrum is partitioned into $K$ groups with a constant number of bands, then borders of each group are iteratively reestimated in order to minimize the internal variance $\sigma_{I}^{2}$ of each group:

$$
\sigma_{I}^{2}(k)=\frac{1}{K} \sum_{k=0}^{K-1} I^{2}\left(z_{k}, z_{k+1}\right)-\left(\frac{1}{K} \sum_{k=0}^{K-1} I\left(z_{k}, z_{k+1}\right)\right)^{2},
$$

where $z_{k}$ represents the position of the upper boundary of the $k^{t h}$ group. In skin images various objects like hair, lips, or eyes can be present. The presence of these objects influences the physics of the spectrum and, thus, the classification. Therefore, an algorithm that permits a spectral partitioning with a variable number of groups $K$ is needed. The solution proposed below is deduced from the discrete function $F_{I}$ defined as follows:

$$
F_{I}(k)=I\left(b_{k}, b_{k+1}\right), k=1, \ldots, N_{b}-1,
$$

where $b_{k}$ represent spectral bands and $N_{b}$ the number of spectral bands in the initial data. Local maxima of $F_{I}$ give possible borders $z_{k}$ for the spectral partitioning. To choose only significant maxima one can use, e.g., multi-scale analysis or the integral of $F_{I}$ between consecutive maxima. Such an analysis of $F_{I}$ introduces a new parameter which can be the scale step for the multi-scale analysis or a threshold for the $F_{I}$ integral analysis.

\subsection{SVM}

SVM, originally proposed in [9], is a supervised method for data classification. It can be decomposed in two steps. The first one, called the learning step, consists in computing an optimal linear separator between learning classes. This is done by quadratic optimization. When data are not linearly separable, the introduction of a kernel transforms the data space into a higher dimensional space where a linear separator can be computed. The second step consists in classifying the whole data set by evaluating for each pixel the distance to the separator. Methods and algorithms for SVMs are available in $[3,10]$. For the skin analysis, we use a two-class non linear SVM with a Gaussian kernel defined as follows:

$$
K\left(x_{i}, x_{j}\right)=\exp \left(-\frac{\left\|x_{i}-x_{j}\right\|^{2}}{2 \sigma^{2}}\right),
$$

where $x_{i}$ and $x_{j}$ are pixels from the training set.

\section{UNMIXING METHOD}

Unmixing or blind source-separation is a well known technique for analyzing multichannel data. It assumes that the data consists of pure signals, called components, that are linearly mixed. In the skin hyper-pigmentation analysis, one looks for a component (or source) as representative as possible of the melanin concentration. If $X_{i, j}$ denotes the initial data at position $(i, j), A$ the mixture matrix, $S_{i, j}$ the weight of each component vector for the pixel $(i, j)$ and $N_{i, j}$ a vector of 

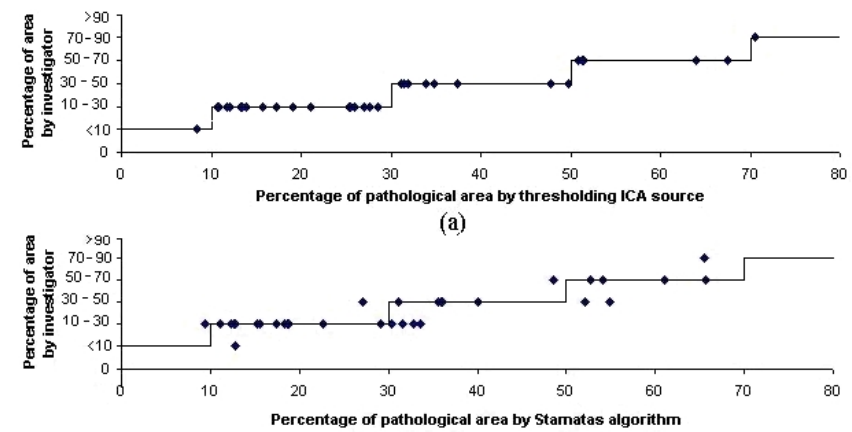

(b)

Fig. 1. Comparaison between dermatologist evaluation of pathological area and detected pathological area by ICA source classification (a) and Stamatas algorithm (b).

additive noise, then the mixing model can be written [11, 12] as:

$$
X_{i, j}=A S_{i, j}+N_{i, j} .
$$

This equation reveals two kinds of ambiguities: when columns of $A$ are transposed or multiplied by different scalars, a new component decomposition is obtained. To estimate sources or independent components, several methods have been proposed in the literature. Here we consider FastICA [12] and Joint Approximate Diagonalization of Eigenmatrices (JADE) [11]. FastICA iteratively computes each component maximizing the negentropy $J$ which is the difference between the entropy $H$ of the source $s$ and that of a Gaussian random variable $g$ with the same correlation matrix as $s$, i.e. $J(s)=H(g)-H(s)$. JADE algorithm performs simultaneous component estimation by diagonalizing matrices of second and fourth order cumulants [11]. Although these two methods are formulated differently, they are close from a mathematical point of view $[11,12]$. That explains why, on our data set, they report comparable results. For JADE algorithm, the more components are estimated, the more memory is needed. FastICA is very sensitive to the model ambiguities, and reports computation time varying from ten seconds to several minutes on different images of the data set. In our case, we look for a small number of sources and prefer to use JADE which has a stable computation time about 20 seconds. Once the most representative source of skin pigmentation is chosen, thresholding enables classification between healthy and hyper-pigmented skin.

\section{EXPERIMENTS}

In this section we compare the developed algorithms with a reference (i.e Stamatas algorithm [1]). These algorithms need manual parametric specification. Projection pursuit combined with SVM (PP-SVM) classification is initialized by choosing training pixels on each image, and melanin concentration cartographies estimated by ICA and Stamatas algorithm are man- ually thresholded to obtain classification. These thresholds are chosen on each image to optimize the correspondence of detected pathological area with a dermatologist analysis.

The two methods, PP-SVM and thresholding a selected ICA component, have been tested on a data set of 96 images taken from 48 patients. These multi-spectral images were provided by Galderma R\&D. These images contain 18 spectral bands in a range from $405 \mathrm{~nm}$ to $970 \mathrm{~nm}$ with an average step of $25 \mathrm{~nm}$. The spatial area is about 900*1200 pixels. On the whole dataset, the algorithms (PP-SVM and ICA) and the ground truth provided by dermatologists report the same pathological area in about $75 \%$ of the images. When algorithms and dermatologist analysis disagreed, it is due to false detections caused by facial features, like lips or eyes, and hair. To compare the accuracy of the skin analysis algorithms, we reduce the data set to 34 images to keep only images that contain as few facial features and hair as possible. In figure 2, we compare results of PP-SVM, thresholding of a selected ICA component and the reference Stamatas algorithm. One can see that on the two images, the three algorithms provide quite similar results. But the Stamatas algorithm fails to detect pathological areas when the contrast between healthy and hyper-pigmented skin is weak. PP-SVM and ICA methods provide better results since they succeed in detecting hyperpigmentation in weak contrasted areas. For example, on the image 1 (left column) of figure 2, ICA based algorithm detects more accurately the weak hyper-pigmented area. Since the PP-SVM initialisation is operator dependent, we demonstrate here only the results for ICA based method. In figure 1(a), we show the correspondence between the dermatologist estimation (quantized into 6 categories by the percentage of hyper-pigmentation), and the detected pathological area by the ICA approch; in figure 1(b), we provide the correspondence with the Stamatas algorithm. Dermatologist analysis and algorithms coincide when dots are on the diagonal line. Comparing with the classification into the 6 classes provided by dermatologists, we obtain $100 \%$ of good classification with the ICA approach whereas Stamatas approach gives $71 \%$ of accurate classification.

\section{CONCLUSION}

In this paper, we have shown that using the whole spectrum instead of only few bands of interest can improve the skin hyper-pigmentation classification on multispectral images. We notice that the developed algorithms have an average computation time of 15 seconds on an image of 900 by 1200 by 18 pixels (with a Matlab code on a $2.2 \mathrm{GHz}$ core processor system) which is a reasonable time for a medical application. In future work, the goal will be to continue using multi/hyperspectral images to provide precise local as well as global skin pigmentation evaluation. Methods to compensate artifacts caused by facial features and hair will be studied. 

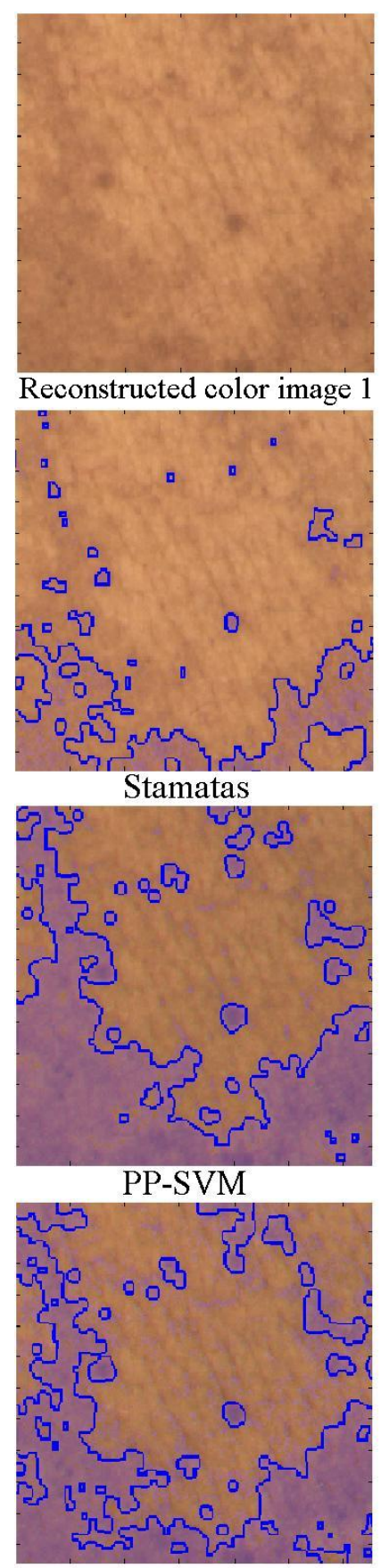

ICA

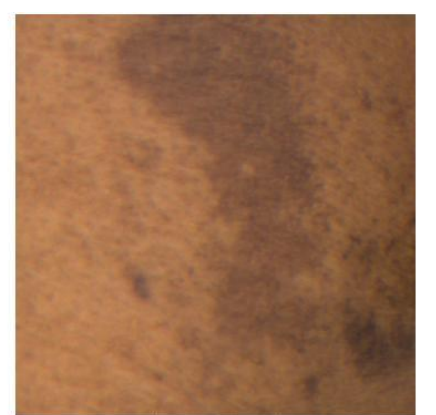

Reconstructed color image 2

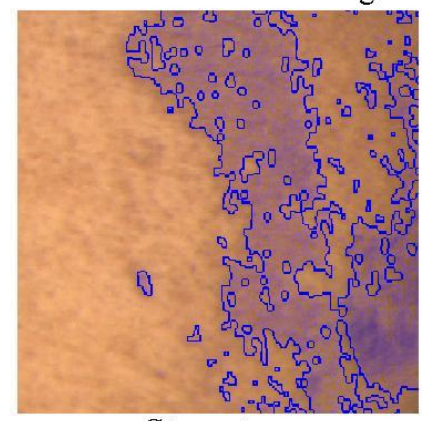

Stamatas

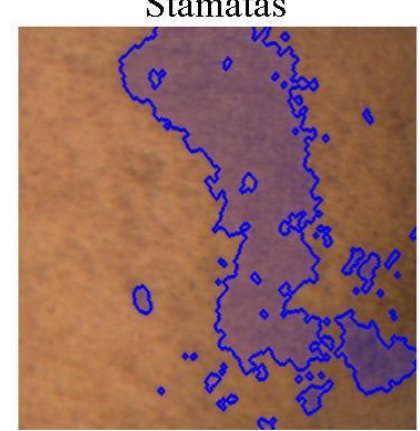

PP-SVM

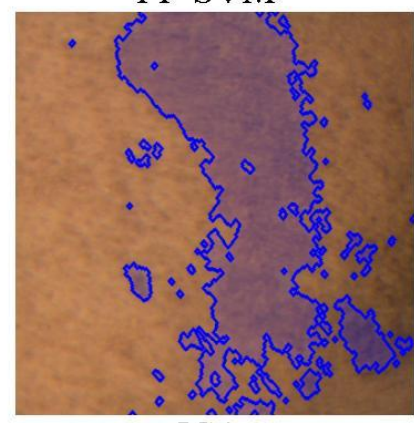

ICA

Fig. 2. Detected pathological areas by Stamatas algorithm, by SVM with Projection Pursuit (PP-SVM), and by thresholding the selected ICA component.

\section{ACKNOWLEDGEMENT}

The authors would like to thank Prof. Jocelyn Chanussot, Dr. Laurent Petit and Anne-Sophie Dugaret for interesting discussions, and Galderma R\&D early development for partial funding and for providing the data.

\section{REFERENCES}

[1] G. N. Stamatas, B. Z. Zmudzka, N. Kollias, and J. Z. Beer, "Non-invasive measurements of skin pigmentation in situ.," Pigment cell res, vol. 17, pp. 618-626, 2004.

[2] G. N. Stamatas, B. Z. Zmudzka, N. Kollias, and J. Z. Beer, "In vivo measurement of skin erythema and pigmentation: new means of implementation of diffuse reflectance spectroscopy with a commercial instrument," British Journal of Dermatology, vol. 159, pp. 683-690, 2008.

[3] M. Fauvel, "Spectral and spatial methods for the classification of urban remote sensing data," in PhD Thesis. Institut National Polytechnique de Grenoble. France, 2007.

[4] S. Mallat and Z. Zhang, "Matching pursuit with timefrequency dictionaries," IEEE Trans. on Signal Processing, vol. 41, pp. 3397-3415, 1993.

[5] G. F. Hughes, "On the mean accuracy of statistical pattern recognizers," IEEE Trans. on Information Theory, vol. 14, pp. 55-63, 1968.

[6] L.O. Jimenez and D.A Landgrebe, "Hyperspectral data analysis and supervised feature reduction via projection pursuit," IEEE Trans. on Geoscience and Remote Sensing, vol. 37, pp. 2653-2667, 1999.

[7] G. Rellier, X. Descombes, F. Falzon, and J. Zerubia, "Texture feature analysis using a gauss-markov model in hyperspectral image classification," IEEE Trans. on Geoscience and Remote Sensing, vol. 42, pp. 15431551, 2004.

[8] D. Gomez, L. K. Harder, B. Ersbll, and J. M. Carstensen, "Precise acquisition and unsupervised segmentation of multi-spectral images," Computer Vision and Image Understanding, vol. 106, pp. 183-193, 2007.

[9] V. Vapnik, "Statistical learning theory," John Wiley and sons, inc., 1998.

[10] O. Zammit, X. Descombes, and J. Zerubia, "Burnt area mapping using support vector machines," International Conference on Forest Fire Research, Figueira da Foz, Portugal, 2006.

[11] J.F. Cardoso, "High-order contrasts for independent component analysis," Neural Computation, vol. 11, pp. 157-192, 1999.

[12] A. Hyvarinen, "Fast and robust fixed-point algorithms for independent component analysis," IEEE Trans. on Neural Networks, vol. 10, pp. 626-634, 1999. 